\title{
Cyclosporin A promotes in vivo myogenic response in collagen VI-deficient myopathic mice
}

\author{
Francesca Gattazzo ${ }^{1,2}$, Sibilla Molon ${ }^{1}$, Valeria Morbidoni ${ }^{1}$, Paola Braghetta ${ }^{1}$, Bert Blaauw ${ }^{3}$, \\ Anna Urciuolo ${ }^{1 * t}$ and Paolo Bonaldo ${ }^{1 *}$
}

1 Department of Molecular Medicine, University of Padova, Padova, Italy

2 Interdepartmental Research Center E. Piaggio, University of Pisa, Pisa, Italy

${ }^{3}$ Department of Biomedical Sciences, University of Padova, Padova, Italy

Edited by:

Emanuele Marzetti, Catholic

University of the Sacred Heart, Italy

Reviewed by:

Urs Ruegg, University of Geneva,

Switzerland

Antonio Musarò, Sapienza University

of Rome, Italy

${ }^{*}$ Correspondence:

Anna Urciuolo and Paolo Bonaldo, Department of Molecular Medicine,

University of Padova, Via U. Bassi

58/B, Padova I-35131, Italy

e-mail:a.urciuolo@ucl.ac.uk;

bonaldo@bio.unipd.it

${ }^{\dagger}$ Present address:

Anna Urciuolo, Stem Cells and Regenerative Medicine, University College London Institute of Child Health, London, UK
Mutations of genes encoding for collagen $\mathrm{VI}$ cause various muscle diseases in humans, including Bethlem myopathy and Ullrich congenital muscular dystrophy. Collagen VI null $\left(\right.$ Col6a $\left.1^{-l-}\right)$ mice are affected by a myopathic phenotype with mitochondrial dysfunction, spontaneous apoptosis of muscle fibers, and defective autophagy. Moreover, Col6a $1^{-1-}$ mice display impaired muscle regeneration and defective self-renewal of satellite cells after injury. Treatment with cyclosporin A (CsA) is effective in normalizing the mitochondrial, apoptotic, and autophagic defects of myofibers in Col6a $1^{-1-}$ mice. A pilot clinical trial with CsA in Ullrich patients suggested that CsA may increase the number of regenerating myofibers. Here, we report the effects of CsA administration at $5 \mathrm{mg} / \mathrm{kg}$ body weight every $12 \mathrm{~h}$ in Col6a $1^{-1-}$ mice on muscle regeneration under physiological conditions and after cardiotoxin (CdTx)-induced muscle injury. Our findings indicate that CsA influences satellite cell activity and triggers the formation of regenerating fibers in Col6a $1^{-/}$mice. Data obtained on injured muscles show that under appropriate administration, regimens CsA is able to stimulate myogenesis in Col6a1 $1^{-1-}$ mice by significantly increasing the number of myogenin (MyoG)-positive cells and of regenerating myofibers at the early stages of muscle regeneration. CsA is also able to ameliorate muscle regeneration of Col6a $1^{-1-}$ mice subjected to multiple CdTx injuries, with a concurrent maintenance of the satellite cell pool. Our data show that CsA is beneficial for muscle regeneration in Col6a $1^{-1-}$ mice.

Keywords: collagen VI, skeletal muscle, congenital muscular dystrophy, animal model, cyclosporin A, muscle regeneration

\section{INTRODUCTION}

Collagen VI is an extracellular matrix protein that forms a microfibrillar network in the endomysium of skeletal muscle. The critical role played by this protein in muscle is clearly shown by collagen VI null (Col6a1 $\left.{ }^{-1-}\right)$ mice, which display an early onset myopathic phenotype characterized by mitochondrial dysfunction, defective autophagy, and spontaneous apoptosis of muscle fibers (Bonaldo et al., 1998; Irwin et al., 2003; Grumati et al., 2010). Cyclosporin A (CsA) is a well-known immunosuppressant drug that was found to have multiple beneficial effects on the myopathic phenotype of Colbat ${ }^{-1-}$ mice, including (i) decreased opening of the mitochondrial permeability transition pore; (ii) rescue of myofiber apoptosis; (iii) stimulation of autophagy in muscle fibers; and (iv) recovery of muscle strength (Irwin et al., 2003; Grumati et al., 2010). Mutations of COL6 genes in humans cause several muscle disorders, including Bethlem myopathy and Ullrich congenital muscular dystrophy (Lampe and Bushby, 2005). A pilot clinical trial in Ullrich and Bethlem patients showed that CsA favorably affects mitochondrial function and dramatically decreases the incidence of apoptosis in muscle fibers. Notably, a significant increase in the number of regenerating myofibers was observed in younger patients undergoing CsA treatment, suggesting that CsA may also increase the overall efficiency of muscle regeneration in patients (Merlini et al., 2008, 2011).

Muscle regeneration relies on the presence of satellite cells, which are quiescent under physiological conditions but become activated upon damage, thus undergoing proliferation and terminal differentiation. At the same time, a subset of activated satellite cells returns to the quiescent state in their original niche under the basal lamina, through a self-renewal process (Tedesco et al., 2010). The differentiation of satellite cells is regulated by a number of transcription factors, where Pax7 is required for satellite cell specification and survival, whereas MyoD, myogenin (MyoG), and MRF4 are essential for satellite cell proliferation and differentiation (Buckingham and Rigby, 2014). Terminal differentiation coincides with the abundant synthesis of myosin heavy chain (MHC). The cardiotoxin (CdTx) injury model is widely used to investigate skeletal muscle regeneration (Chargé and Rudnicki, 2004; Shi and Garry, 2006). We recently demonstrated that collagen VI is a critical component of satellite cell niche and that ablation of collagen VI leads to impaired muscle regeneration and reduced satellite cell self-renewal after injury (Urciuolo et al., 2013). Studies performed in tibialis anterior (TA) muscle showed that Colba1 ${ }^{-1-}$ mice undergo a marked depletion of the satellite cell pool 7 days after CdTx injection, and this defect 
becomes much more dramatic after multiple rounds of CdTx injury (Urciuolo et al., 2013). Given the effects displayed by CsA in collagen VI-deficient mice and Ullrich/Bethlem patients (Irwin et al., 2003; Merlini et al., 2008), here we analyzed in detail the outcomes of CsA administration on muscle regeneration and satellite cells in Col6a1 $1^{-1-}$ mice under physiological conditions and after CdTx-induced injury.

\section{MATERIALS AND METHODS \\ MICE}

We performed experiments in wild-type mice of the inbred $\mathrm{C} 57 \mathrm{BL} / 6 \mathrm{NCrl}$ strain and in Col6a1 $1^{-1-}$ mice that were backcrossed in the C57BL/6NCrl strain for eight generations (Irwin et al., 2003). All data were obtained from 6-month-old mice. Mice were housed in individual cages in an environmentally controlled room $\left(23^{\circ} \mathrm{C}, 12 \mathrm{~h}\right.$ light $/ 2 \mathrm{~h}$ dark cycle) and provided food and water ad libitum. Mouse procedures were approved by the Ethics Committee of the University of Padova and authorized by the Italian Ministry of Health.

\section{IN VIVO TREATMENTS}

Cyclosporin A (Sandimmun $50 \mathrm{mg} / \mathrm{ml}$, Novartis) was dissolved in olive oil and a stock solution at a concentration of $10 \mathrm{mg} / \mathrm{ml}$ was prepared. For CsA administration under physiological conditions, mice were subjected to intraperitoneal (i.p.) injection of vehicle (olive oil) or CsA at $5 \mathrm{mg} / \mathrm{kg}$ body weight every $12 \mathrm{~h}$ for 10 days. In experiments with higher dosage CsA, mice were subjected to i.p. injection of vehicle or CsA at $25 \mathrm{mg} / \mathrm{kg}$ body weight every $24 \mathrm{~h}$ for 10 days. Animals were sacrificed $12 \mathrm{~h}$ after the last administration of CsA or vehicle. For single CdTx injury (Couteaux et al., 1988), mice were treated by i.p. injection with vehicle or CsA at $5 \mathrm{mg} / \mathrm{kg}$ body weight every $12 \mathrm{~h}$ for 10 days. At day 4 from the first administration of vehicle or CsA, mice were anesthetized with isoflurane (Merial) and TA muscles injected with $30 \mu \mathrm{l} \mathrm{CdTx}$ (Naja mossambica mossambica, $10 \mu \mathrm{M}$; Sigma). Analgesia (Rimadyl) was administered subcutaneously for 3 days and mice were sacrificed 7 days after muscle damage (i.e., 10 days after the first injection of vehicle or CsA). For multiple injury experiments, TA muscles were subjected to three distinct injections of CdTx, each one every 30 days. Four days before the third CdTx injection, mice were treated by i.p. injection with vehicle or CsA at $5 \mathrm{mg} / \mathrm{kg}$ body weight every $12 \mathrm{~h}$ for 10 days. Mice were sacrificed 30 days after the third CdTx injury (i.e., 24 days after the last injection of vehicle or CsA).

\section{HISTOLOGICAL ANALYSIS}

Tibialis anterior muscles were isolated from mice, frozen in liquid nitrogen, weighted on a precision balance, and kept at $-80^{\circ} \mathrm{C}$ until use. Cross-sections ( $10 \mu \mathrm{m}$ thick) were used and processed for hematoxylin-eosin or Azan-Mallory staining following standard protocols. Samples were analyzed with a Zeiss Axioplan light microscope equipped with Leica DC500 digital camera. Myofiber cross-sectional area and the area of fibrosis were evaluated with the IM1000 software (Leica).

\section{ISOLATION OF EXTENSOR DIGITORUM LONGUS SINGLE MYOFIBERS}

We carefully dissected extensor digitorum longus (EDL) muscles from 6-month-old mice and subjected them to enzymatic digestion with collagenase I ( $2 \mathrm{mg} / \mathrm{ml}$, Gibco) for $80 \mathrm{~min}$ at $37^{\circ} \mathrm{C}$. We blocked the digestion with Dulbecco's Modified Eagle Medium (DMEM, Sigma), supplemented with $0.2 \mathrm{M} \mathrm{L}^{-}$ glutamine (Invitrogen), 1:100 penicillin-streptomycin (Invitrogen), 1:100 fungizone (Invitrogen), and 10\% horse serum (Gibco), and gently released single myofibers from muscles. Every 15$25 \mathrm{~min}$, undamaged and non-contracted fibers were transferred in a new dish containing fresh medium, and this procedure was repeated five times in order to remove debris and interstitial cells. Freshly isolated fibers were finally fixed in $4 \%$ paraformaldehyde in PBS for $15 \mathrm{~min}$ and maintained at $4^{\circ} \mathrm{C}$ in PBS until use.

\section{IMMUNOFLUORESCENCE}

For immunofluorescence on muscle sections, frozen TA sections $(7 \mu \mathrm{m})$ were fixed for $20 \mathrm{~min}$ with $4 \%$ paraformaldehyde in PBS and permeabilized for $6 \mathrm{~min}$ with cold methanol. For the unmasking of Pax7 and MyoG, slides were treated twice with $0.01 \mathrm{M}$ citric acid ( $\mathrm{pH} \mathrm{6)}$ at $90^{\circ} \mathrm{C}$ for $5 \mathrm{~min}$. For mouse antibodies staining, samples were first incubated for $2.5 \mathrm{~h}$ with $4 \%$ bovine serum albumin (BSA IgG-Free, Jackson Immunoresearch) in PBS and then treated for $30 \mathrm{~min}$ with a blocking solution containing $0.05 \mathrm{mg} / \mathrm{ml}$ Fab fragment anti-mouse IgG (Jackson Immunoresearch). When mouse antibodies were not used, samples were only incubated for $1 \mathrm{~h}$ at room temperature with $4 \%$ bovine serum albumin in PBS. After the blocking step, samples were incubated with primary antibodies at $4^{\circ} \mathrm{C}$ overnight. The following primary antibodies were used: mouse anti-Pax7 (1:20; Developmental Studies Hybridoma Bank); mouse antiMyoG (F5D, 1:15; Developmental Studies Hybridoma Bank); mouse anti-embryonic MHC (eMHC) (F1.652, 1:20; Developmental Studies Hybridoma Bank); rabbit anti-laminin (L9393, 1:800; Sigma). After washing, samples were incubated with the appropriate secondary antibody for $1 \mathrm{~h}$ at room temperature. Secondary antibodies used were biotinylated anti-mouse (115007-003, 1:1000), Cy2 or Cy3 anti-mouse (115-226-062, 1:500, or 115-165-006, 1:1000), Cy2 or Cy3 anti-rabbit (111-225-144, 1:500, or 115-165-006, 1:1000) (all Jackson Immunoresearch). To reveal the biotinylated antibody, Cy2 or Cy3 streptavidin (016-220084, 1:1500, or 016-160-084, 1:2500; Jackson Immunoresearch) was used. For immunofluorescence of EDL single myofibers, cells were permeabilized with $0.5 \%$ Triton X-100 in PBS, treated with $20 \%$ goat serum (Invitrogen) in PBS for $1 \mathrm{~h}$, and incubated at $37^{\circ} \mathrm{C}$ for $1 \mathrm{~h}$ or at $4^{\circ} \mathrm{C}$ overnight with mouse anti-Pax7 antibody (1:20; Developmental Studies Hybridoma Bank). After washing, samples were incubated with the appropriate secondary antibody as described above. Nuclei were stained with Hoechst 33258 (Sigma). Samples were analyzed with a Zeiss Axioplan Leica DC500 epifluorescence microscope or with a Leica SP5 confocal microscope.

\section{TUNEL}

For apoptosis analysis on TA cryosections, the DeadEnd ${ }^{\mathrm{TM}}$ Fluorometric TUNEL assay (Promega) was used. Samples were fixed for $15 \mathrm{~min}$ with $4 \%$ paraformaldehyde, permeabilized for $5 \mathrm{~min}$ with $0.5 \%$ Triton X-100, and processed following manufacturer instructions. 

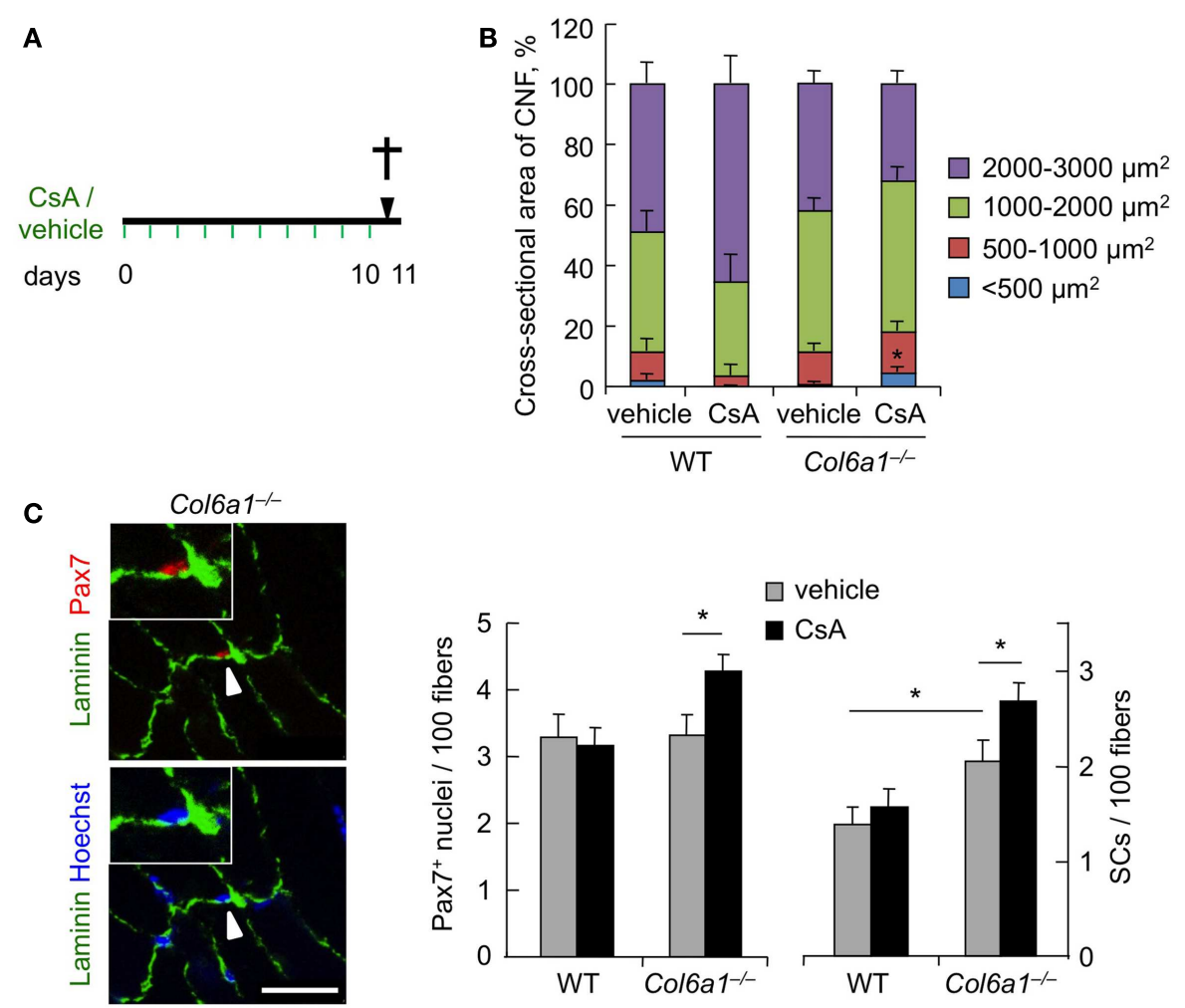

FIGURE 1 | Cyclosporin A induces muscle regeneration and increases satellite cell number in Col6a 1-l- mice. (A) Schematic diagram of CsA treatment. Wild-type and Col6a1 $1^{-1-}$ mice were treated with vehicle or with CsA ( $5 \mathrm{mg} / \mathrm{kg}$ body weight) every $12 \mathrm{~h}$ for 10 days. Animals were sacrificed $12 \mathrm{~h}$ after the last administration of CsA or vehicle. (B) Mean cross-sectional area of regenerating centrally nucleated myofibers in TA muscles derived from wild-type and Col6a $1^{-1-}$ mice treated with vehicle or CsA. Fibers were divided into four size ranges, and at least 150 centrally nucleated myofibers were analyzed for each group. Error bars indicate SEM ( ${ }^{*} P<0.05$ for Col6a1 ${ }^{-1-}$ CsA vs. Col6a $1^{-1-}$ vehicle; $n=3-4$, each group). (C) Left panel, representative images of immunofluorescence labeling for laminin (green) and $\mathrm{Pax} 7$ (red) in TA cross-sections of CsA-treated Col6a $1^{-1-}$ mice. The arrowhead points at one satellite cell, shown at higher magnification in the inset. Nuclei were stained with Hoechst (blue). Scale bar, $25 \mu \mathrm{m}$. Right panel, quantification of total Pax7-positive cells and of satellite cells, calculated as the number on 100 myofibers in TA muscles derived from wild-type and Col6a $1^{-1-}$ mice treated with vehicle or CSA. Error bars indicate SEM ${ }^{*} P<0.05 ; n=5-7$, each group). CNF, centrally nucleated fibers; CSA, cross-sectional area; SCs, satellite cells; WT, wild-type.

\section{STATISTICAL ANALYSES}

Data are expressed as means \pm SEM. We determined statistical significance by unequal variance Student's $t$-test, and a $P$ value of $<0.05$ was considered statistically significant.

\section{RESULTS}

\section{CsA INDUCES MUSCLE REGENERATION IN Col6a1-/- MICE UNDER PHYSIOLOGICAL CONDITIONS}

To investigate the effects of CsA administration in Col6a $1^{-1-}$ mice under physiological conditions, we subjected animals to i.p. injection of vehicle or CsA at $5 \mathrm{mg} / \mathrm{kg}$ body weight every $12 \mathrm{~h}$ and analyzed muscles after 10 days of treatment (Figure 1A). This dosage of CsA was previously found to trigger a marked amelioration of the myopathic phenotype of Col6a1 ${ }^{-1-}$ mice, with rescue from mitochondrial depolarization and apoptosis and reactivation of the autophagic flux in muscle fibers (Irwin et al., 2003; Grumati et al., 2010). To evaluate whether this CsA treatment triggered de novo formation of myofibers in Col6a1 $1^{-1-}$ mice, we first analyzed the cross-sectional area of regenerating, centrally nucleated fibers in TA muscle, by dividing regenerating myofibers into four different size ranges. Unlike wild-type animals, CsA treatment led to a significant increase of the percentage of regenerating myofibers with small area $\left(<500 \mu \mathrm{m}^{2}\right)$ in Col6a1 ${ }^{-1-}$ mice when compared to vehicle-treated Col6a1 $1^{-1-}$ animals (Figure 1B). These data were also confirmed by immunofluorescence analysis for eMHC, an established marker of newly forming fibers (Ciciliot and Schiaffino, 2010). Immature myofibers expressing eMHC were present in TA muscles of Col6a1 $1^{-1-}$ mice treated with CsA, but not in those treated with vehicle (Figure S1A in Supplementary Material). Based on these results, we evaluated the number of myogenic cells by performing immunostaining for Pax7. CsA administration increased the total number of Pax7-positive cells and also the number of satellite cells (i.e., Pax7-positive cells located underneath the basal lamina) in Col6a1 ${ }^{-1-}$ TA but not in wild-type TA (Figure 1C). These data were confirmed by analyzing freshly isolated EDL myofibers, which showed a significant increase in the number of Pax7-positive cells on myofibers derived from CsAtreated Col6a1 ${ }^{-1-}$ mice when compared to vehicle-treated animals (Figure S1B in Supplementary Material). To assess whether the increased number of Pax7-positive cells in Col6a1 $1^{-1-}$ animals was 

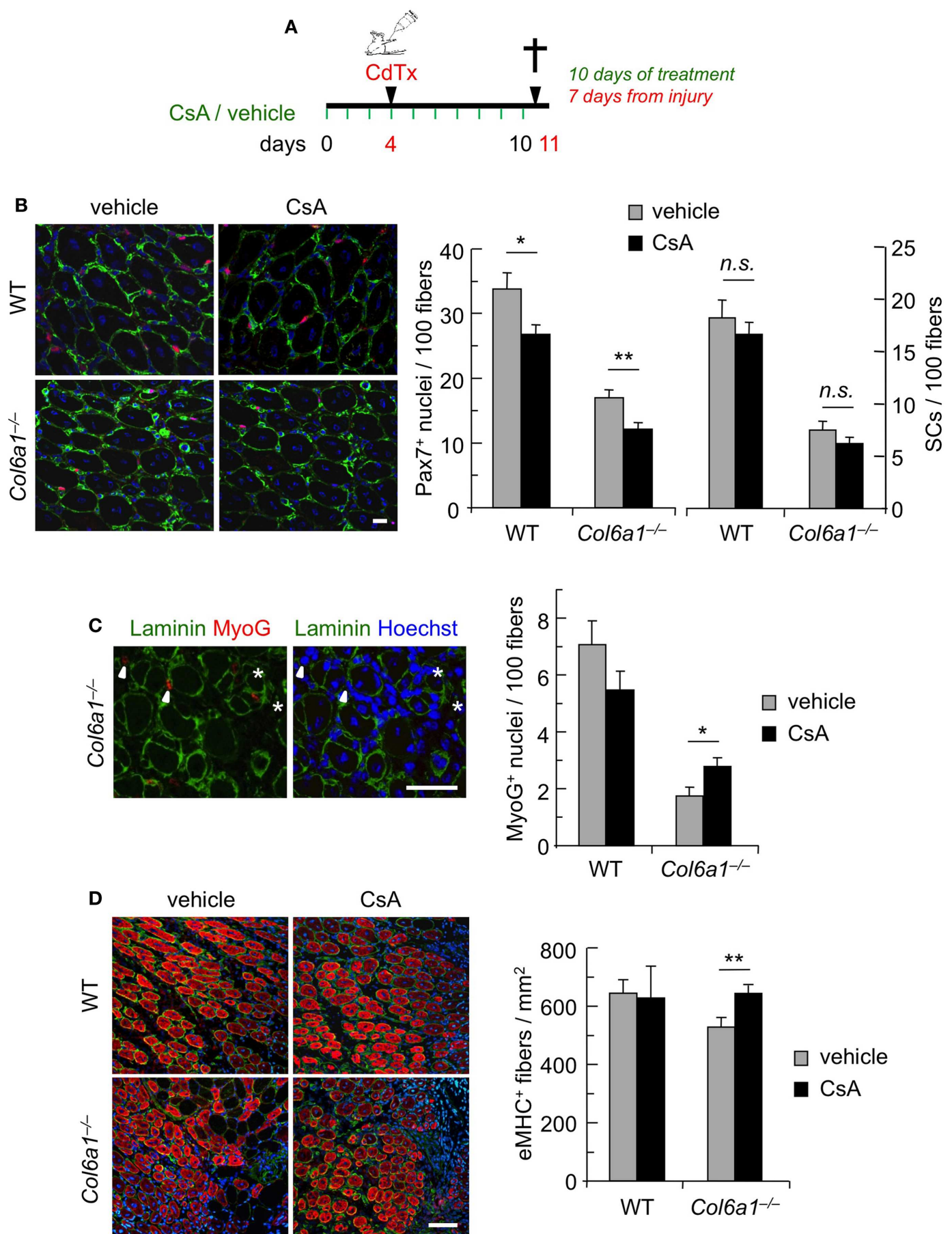

FIGURE 2 | Continuous administration of CsA during a single CdTx injury stimulates muscle differentiation in Col6a1 ${ }^{-1-}$ mice.

(A) Schematic diagram of CsA treatment and CdTx injury. Wild-type and Col6a $1^{-1-}$ mice were treated with vehicle or CsA ( $5 \mathrm{mg} / \mathrm{kg}$ body weight) every $12 \mathrm{~h}$ for 10 days. Four days after the first injection of vehicle or CsA, TA muscles were injured with $\mathrm{CdTx}$ and drug treatment continued for other 7 days. Animals were sacrificed $12 \mathrm{~h}$ from the last administration of CsA or vehicle. (B) Left panels, representative images of immunofluorescence labeling for laminin (green) and $\mathrm{Pax} 7$ (red) in 7-day-post-injury TA cross-sections of wild-type and Col6a $1^{-1-}$ mice treated with vehicle or CsA. Scale bar, $25 \mu \mathrm{m}$. Right panel, quantification of total Pax7-positive cells and of satellite cells, calculated as the number on 100 myofibers in 7-day-post-injury TA muscles of wild-type and Col6a $1^{-1-}$ mice treated with vehicle or CsA. Error bars indicate SEM ( ${ }^{*} P<0.01$; ${ }^{*} P<0.05$; n.s. not significant; $n=3-5$, each group). (C) Left panels, representative images of immunofluorescence labeling for laminin (green) and MyoG (red) in 7-day-post-injury TA cross-sections of Col6a 1-1mice treated with vehicle or CsA. Nuclei were stained with Hoechst (blue). The arrowheads point at MyoG-positive cells outside the basal lamina, and the asterisks mark MyoG-positive cells located underneath the basal lamina (corresponding to myogenic cells that are undergoing fusion). 


\section{FIGURE 2 | Continued}

Scale bar, $50 \mu \mathrm{m}$. Right panel, quantification of total MyoG-positive cells, calculated as the number on 100 myofibers in 7-day-post-injury TA muscles of wild-type and Col6a $1^{-1-}$ mice treated with vehicle or CsA. Error bars indicate SEM ( ${ }^{*} P<0.05 ; n=3-5$, each group). (D) Left panels, representative images of immunofluorescence for laminin (green) and $\mathrm{eMHC}$ (red) in 7-day-post-injury TA cross-sections of wild-type and Col6a1 $1^{-1-}$ mice treated with vehicle or CsA. Nuclei were stained with Hoechst (blue). Scale bar, $50 \mu \mathrm{m}$. Right panel, quantification of the number of eMHC-positive myofibers per regenerating area in 7-day-post-injury TA cross-sections of wild-type and Col6a $1^{-1-}$ mice treated with vehicle or CsA. Error bars indicate SEM ( ${ }^{*} P<0.01 ; n=3-5$, each group). SCs, satellite cells; WT, wild-type. only provided by this drug dosage or could be also elicited by higher CsA concentrations known to cause strong immunosuppressive effects (Homan et al., 1980), we subjected mice to i.p. injection of CsA at $25 \mathrm{mg} / \mathrm{kg}$ body weight every $24 \mathrm{~h}$ for 10 days. Interestingly, at this higher dosage, CsA led to a dramatic decrease in the number of total Pax7-positive cells and of satellite cells both in wild-type and in Col6a1 $1^{-1-}$ mice when compared to vehicletreated animals (Figure S1C in Supplementary Material). These results highlight the relevance of CsA dosage in inducing beneficial effects in Col6a1 $1^{-/-}$muscles and accordingly with previous studies carried out with the immunosuppressive drug FK506 (Irwin et al., 2003), they suggest that immunosuppression exacerbates the phenotype of Col6a1-1- mice.

\section{CONTINUOUS ADMINISTRATION OF CSA DURING CDTX INJURY STIMULATES THE EARLY PHASES OF MUSCLE DIFFERENTIATION IN Col6a1 $^{-1-}$ MICE}

To further assess the capability of CsA to ameliorate the muscle regenerative defects of Col6a1 $1^{-1-}$ mice, we carried out CsA treatment under experimentally induced muscle injury. Toward this aim, wild-type and Col6a1 $1^{-1-}$ mice were treated for 10 days with vehicle or CsA at $5 \mathrm{mg} / \mathrm{kg}$ body weight every $12 \mathrm{~h} ; 4$ days after the start of treatment, TA muscles were subjected to CdTx damage and mice were sacrificed 7 days after injury (Figure 2A). TUNEL assay showed that the incidence of apoptotic nuclei 7 days after CdTx injection was very low in wild-type TA, in agreement with the concept that myofiber demise is almost completed at this stage from injury (Hawke et al., 2003). Conversely, Col6a1 ${ }^{-1-}$ TA showed a higher number of TUNEL-positive myonuclei 7 days after injury, and CsA administration was able to significantly decrease the incidence of apoptotic myofibers triggered by CdTx injury in Col6a1 ${ }^{-1-}$ TA muscles (Figure S2 in Supplementary Material). We next evaluated the number of myogenic cells by immunofluorescence for the Pax7 and MyoG markers. CsA administration led to a significant decrease in the number of total Pax7-positive cells in both wild-type and Col6a1-1- injured TA, without any significant change in the number of satellite cells (i.e., Pax7positive cells located underneath basal lamina) (Figure 2B). This response was paralleled by a significant increase of the total number of MyoG-positive cells in injured TA muscles of CsA-treated Col6a1 ${ }^{-1-}$ mice when compared to vehicle-treated Col6a1 ${ }^{-1-}$ mice, whereas wild-type injured TA muscles did not show any significant difference in MyoG positivity between vehicle and CsA-treated animals (Figure 2C). Additionally, Col6a1 ${ }^{-1-}$ mice treated with CsA showed an increased number of regenerating myofibers expressing eMHC, whereas no differences in eMHCpositive regenerating myofibers were found in wild-type animals (Figure 2D). As the defective satellite self-renewal of Col6a1 ${ }^{-1-}$ mice is strictly dependent on the lack of extracellular collagen VI and on the lower muscle stiffness (Urciuolo et al., 2013), it was not surprising to observe that in this experimental condition CsA does not display any overt effect on satellite cell maintenance. On the other hand, the remarkable increase in the number of differentiated (i.e., MyoG-positive) myogenic cells, together with the higher number of newly forming (i.e., eMHC-positive) myofibers, indicates that CsA administration is able to improve muscle differentiation upon damage in the Col6a1 ${ }^{-1-}$ myopathic mouse model.

\section{ADMINISTRATION OF CSA DURING REPEATED MUSCLE INJURY COUNTERACTS MUSCLE LOSS AND FIBROSIS AND PRESERVES THE SATELLITE CELL POOL IN Col6a1-/- MICE}

Although muscles of Col6a1 $1^{-1-}$ animals display a delayed regeneration after injury, we have previously shown that at 30 days after CdTx injury they are still able to complete the regeneration process (Urciuolo et al., 2013). However, and at difference from wild-type mice, the capability of Col6a1 $1^{-1-}$ animals to undergo muscle regeneration and preserve the satellite cell pool is lost after multiple muscle injuries, leading to a severe loss of muscle mass (Urciuolo et al., 2013). Therefore, we investigated whether CsA is able to counteract the defective muscle regeneration and the depletion of the satellite cell pool triggered by multiple injuries in Col6a1 $1^{-1-}$ mice. Toward this aim, we subjected TA muscles of wild-type and Col6a1 $1^{-1-}$ mice to three rounds of CdTx injury. Animals were treated for 10 days with vehicle or CsA at $5 \mathrm{mg} / \mathrm{kg}$ body weight every $12 \mathrm{~h}$ during the third injury and sacrificed 30 days after the last injury (Figure 3A). Interestingly, CsA administration was highly effective in reducing the extensive muscle fibrosis triggered by triple injury in Col6a1 $1^{-1-}$ mice (Figure 3B). The beneficial effects of CsA in Col6a1 ${ }^{-1-}$ muscles undergoing multiple injuries were also confirmed by the increased myofiber cross-sectional area and by the improvement of the muscle mass in CsA-treated Col6a1 $1^{-1-}$ mice when compared to vehicle-treated Col6a1 ${ }^{-1-}$ mice (Figure S3 in Supplementary Material). Notably, CsA administration led to marked increase of both total Pax7positive cell number and satellite cell number in Col6a1 ${ }^{-/-} \mathrm{TA}$ muscles subjected to multiple injuries (Figures 3C,D). Altogether, these results show that at this regimen CsA is capable to preserve not only muscle fibers but also the satellite cell pool of collagen VI-deficient mice.

\section{DISCUSSION}

In the present study, we evaluated the potential beneficial effects exerted by CsA on skeletal muscle regeneration in Col6a1 $1^{-1-}$ mice, both under physiological condition and after muscle damage. The rationale for this study was based on previous findings in patients affected by collagen VI myopathies, suggesting that besides counteracting myofiber apoptosis and mitochondrial dysfunction, CsA treatment may also increase muscle regeneration (Merlini et al., 2008, 2011). In addition, our recent findings 
A

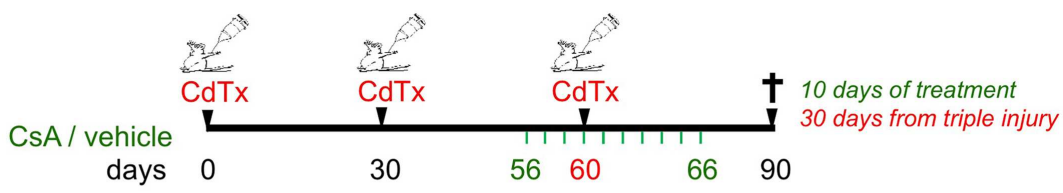

B

vehicle
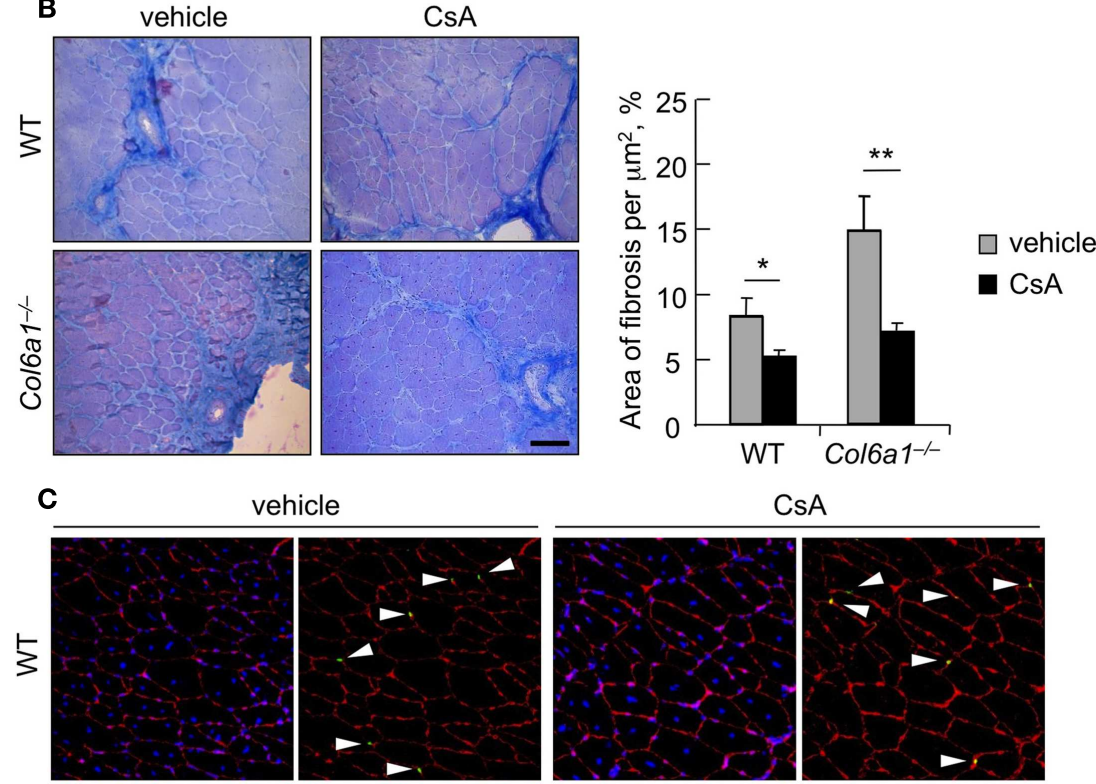

vehicle

CsA
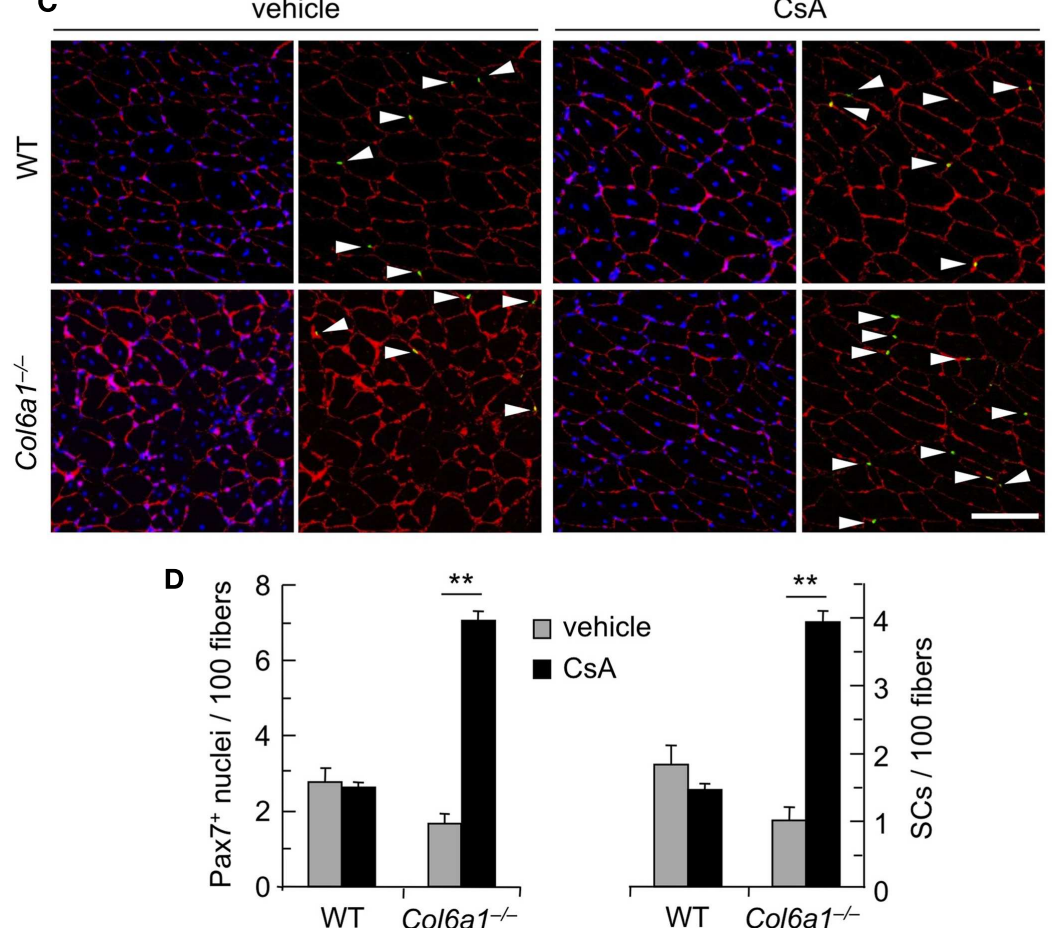

FIGURE 3 | Administration of CsA during a triple muscle injury counteracts muscle loss and fibrosis and preserves the satellite cell pool in Col6a 1-1- mice. (A) Schematic diagram of CsA treatment and $\mathrm{CdTx}$ injuries. TA muscles of wild-type and Col6a1 $1^{-1-}$ mice were given three repeated injections of CdTx every 30 days. Four days before the last injury, Col6a $1^{-1-}$ mice were treated with vehicle or CsA ( $5 \mathrm{mg} / \mathrm{kg}$ body weight) every $12 \mathrm{~h}$ for 10 days. Mice were sacrificed 30 days after the third injury (i.e., 24 days from the last administration of vehicle or CsA). (B) Left panels, Azan-Mallory staining of triple injured TA cross-sections from wild-type and Col6a $1^{-1-}$ mice treated with vehicle or CsA. Scale bar, $50 \mu \mathrm{m}$. Right panel, quantification of the fibrotic area in triple-injured TA cross-sections from

wild-type and Col6a1 $1^{--}$mice treated with vehicle or CsA. Error bars indicate SEM ${ }^{* *} P<0.01 ;{ }^{*} P<0.05 ; n=4-8$, each group).

(C) Representative images of immunofluorescence labeling for laminin (red) and Pax7 (green) in triple-injured TA cross-sections from wild-type and Col6a $1^{-1-}$ treated with vehicle or CsA. Arrows point at Pax7-positive nuclei. Nuclei were stained with Hoechst (blue). Scale bar, $100 \mu \mathrm{m}$.

(D) Quantification of total Pax7-positive cells and of satellite cells, calculated as the number on 100 myofibers in triple-injured TA cross-sections from wild-type and Col6a $1^{-1-}$ mice treated with vehicle or CsA. Error bars indicate SEM ( $^{*} P<0.01 ; n=4-8$, each group). SC, satellite cells; WT, wild-type. showed impaired regeneration and defective satellite cell selfrenewal in collagen VI-deficient muscles (Urciuolo et al., 2013). Therefore, we investigated how CsA treatment impacts on the regeneration of collagen VI-deficient mice. Under physiological conditions, CsA was capable to amplify the pool of total Pax7positive cells and of satellite cells and increased the amount of 
newly formed centrally nucleated myofibers, thus suggesting a new role for CsA in stimulating myogenesis in Col6a1 ${ }^{-1-}$ muscles. Notably, these beneficial effects were dose-dependent, as they were observed at $10 \mathrm{mg} / \mathrm{kg} /$ day but not at a higher immunosuppressant dose $(25 \mathrm{mg} / \mathrm{kg} /$ day $)$, which conversely had a negative impact on the satellite cell pool. These data are in agreement with previous results in which the same protocol of CsA administration was found to desensitize the mitochondrial permeability transition pore and reduce myofiber apoptosis in Col6a1 ${ }^{-1-}$ mice (Irwin et al., 2003). Although we previously demonstrated that CsA stimulates autophagy in skeletal myofibers (Grumati et al., 2010), the increased number of satellite cells in muscles of CsA-treated Col6a1 ${ }^{-1-}$ animals is not a direct consequence of a stimulatory effect on autophagy. Indeed, reactivation of the autophagic flux in Col6a1 $1^{-1-}$ mice by different pharmacological or dietary treatments does not exert any significant effect on satellite cells (Urciuolo et al., 2013). Our present findings are consistent with the strong amelioration of the myophatic phenotype in Col6a1 $1^{-1-}$ mice following CsA administration and indicate that besides decreasing mitochondrial dysfunction and apoptosis and reactivating autophagy in muscle fibers (Irwin et al., 2003; Grumati et al., 2010), the drug is also able to increase the pool of functional Pax7-positive cells and stimulate the formation of newly formed fibers.

The beneficial effects exerted by CsA on the regeneration capabilities of Col6a1 $1^{-1-}$ mice become very evident under experimentally induced single and multiple muscle injuries. Our data indicate that CsA is capable to induce myogenesis in Col6a1 ${ }^{-1-}$ mice after muscle damage. In fact, when TA muscles were damaged during a continuous CsA administration, analysis at 7 days post-injury showed that CsA elicits a significant increase in the number of MyoG-positive cells and of regenerating myofibers in Col6a1 $1^{-1-}$ muscles. Interestingly, this response is not associated with an improvement of the number of satellite cells, suggesting that under these conditions CsA is unable to ameliorate satellite cell self-renewal. The effect of CsA on muscle regeneration was even more remarkable when we exacerbated the muscle phenotype of Col6a1 ${ }^{-1-}$ mice through triple CdTx damage. Our findings indicate that CsA is protective against fibrotic tissue formation, maybe exerting this effect through an indirect regulation of the inflammatory state that occurs during muscle regeneration (Serrano et al., 2011). A similar beneficial effect of CsA in reducing muscle fibrosis was reported for $m d x$ mice undergoing exercise (De Luca et al., 2005). Furthermore, CsA administration was able to counteract the loss of satellite cells elicited by repeated muscle injuries in Col6a1 $1^{-/-}$animals, concurrently guaranteeing myogenic differentiation, as confirmed by the increase of myofiber cross-sectional area and muscle mass. Although it was beyond the scope of this study to dissect the mechanism(s) through which CsA leads to increased satellite cell number in Col6a1 $1^{-1-}$ mice after repeated injuries, it can be hypothesized that CsA administration may not directly influence the self-renewal capability of satellite cells and that the preservation of satellite cell pool may be mediated by an increase of their survival. This assumption is supported by the fact that the defective satellite cell self-renewal of Col6a1 ${ }^{-1-}$ mice is strictly dependent on the lack of collagen VI itself and its consequences on muscle stiffness (Urciuolo et al., 2013) and that
CsA treatment is able to reduce apoptosis in Col6a1 ${ }^{-1-}$ muscles (Irwin et al., 2003). To our knowledge, no literature work has investigated in detail the effects of in vivo CsA administration on stem cell homeostasis in skeletal muscles. A recent study reported some beneficial effects of CsA on neuronal stem cells, showing that in vivo CsA administration increases the number of neurospheres due to enhanced neuronal stem cell survival, rather than increased proliferation (Hunt et al., 2010).

The pharmacology of CsA is complex, and the drug binds a family of cellular peptidyl-prolyl cis-trans isomerases known as cyclophilins. Binding of CsA with the abundant cyclophilin A leads to inhibition of calcineurin, a cytosolic phosphatase found in many cell types, thus preventing dephosphorylation of its substrates (Liu et al., 1991). A number of studies have shown that calcineurin signals are involved in the control of myofiber size, myofiber type, and skeletal muscle regeneration (Schiaffino and Serrano, 2002; Sakuma and Yamaguchi, 2010; Hudson and Price, 2013). Although inhibition of calcineurin was shown to delay muscle regeneration (Sakuma et al., 2003, 2005), literature studies investigating the outcomes of calcineurin inhibition by genetic approaches or by CsA administration in animal models of muscle diseases have produced contrasting results (Stupka et al., 2004; De Luca et al., 2005; Parsons et al., 2007). The reasons for these discrepancies rely upon multiple factors, including the genetic model studied, the dose of the drug, the type of muscle, the duration of treatment, and the route of treatment. For instance, the efficacy of CsA in the $m d x$ mice, an animal model of Duchenne muscular dystrophy, was reported to be dependent on the dosage and length of the treatment (Stupka et al., 2004; De Luca et al., 2005). Notably, the protective effects of CsA in Col6a1 ${ }^{-1-}$ mice do not rely upon calcineurin inhibition, as the same beneficial effects are also displayed by non-immunosuppressive CsA analogs that do not bind calcineurin, such as Debio 025 and NIM811 (Angelin et al., 2007; Zulian et al., 2014), whereas they cannot be mimicked by the calcineurin inhibitor FK506 (Irwin et al., 2003). Although our interest was far from the study of calcineurin activity, in this work we used a definite CsA dosage $(5 \mathrm{mg} / \mathrm{kg}$ every $12 \mathrm{~h}$, i.e., the same dose shown to be effective in rescuing different aspects of the muscle pathology of Col6a1 $1^{-1-}$ mice), and this dosage is known to only partially reduce the activity of calcineurin (Dunn et al., 2002; Michel et al., 2004).

In conclusion, our results indicate that besides the already known beneficial effects of CsA administration in ameliorating the myophatic phenotype of Col6a1 $1^{-1-}$ mice through the rescue from mitochondrial and autophagic dysfunction of muscle fibers, CsA is also capable to stimulate muscle regeneration and preserve the satellite cell pool in this disease model. These findings support and strengthen the increased muscle regeneration observed in Ullrich patients undergoing clinical trial with CsA, pointing at CsA and its non-immunosuppressive derivatives as a promising therapeutic route for this group of inherited muscle diseases.

\section{AUTHOR CONTRIBUTIONS}

Francesca Gattazzo planned and performed in vivo and ex vivo experiments and wrote the paper. Sibilla Molon performed cardiotoxin damage, immunofluorescence, and histology. Valeria Morbidoni performed in vivo satellite cell quantification. Bert 
Blaauw carried out part of the in vivo studies. Paola Braghetta was involved in CsA administration. Anna Urciuolo oversaw the results and interpreted the data. Paolo Bonaldo oversaw the results and wrote the paper. All the authors discussed the results, revised the work, commented on the manuscript, and agreed on the final draft.

\section{ACKNOWLEDGMENTS}

This work was supported by grants from Telethon-Italy (GGP10225 and GGP11082), the Italian Ministry of Education, University and Research (FIRB Strategic Project RBAP11Z3YA_003), and the University of Padova.

\section{SUPPLEMENTARY MATERIAL}

The Supplementary Material for this article can be found online at http://www.frontiersin.org/Journal/10.3389/fnagi.2014.00244/ abstract

\section{REFERENCES}

Angelin, A., Tiepolo, T., Sabatelli, P., Grumati, P., Bergamin, N., Golfieri, C., et al. (2007). Mitochondrial dysfunction in the pathogenesis of Ullrich congenital muscular dystrophy and prospective therapy with cyclosporins. Proc. Natl. Acad. Sci. U.S.A. 104, 991-996. doi:10.1073/pnas.0610270104

Bonaldo, P., Braghetta, P., Zanetti, M., Piccolo, S., Volpin, D., and Bressan, G. M. (1998). Collagen VI deficiency induces early onset myopathy in the mouse: an animal model for Bethlem myopathy. Hum. Mol. Genet. 7, 2135-2140. doi:10.1093/hmg/7.13.2135

Buckingham, M., and Rigby, P. W. J. (2014). Gene regulatory networks and transcriptional mechanisms that control myogenesis. Dev. Cell 28, 225-238. doi:10.1016/j.devcel.2013.12.020

Chargé, S. B. P., and Rudnicki, M. (2004). Cellular and molecular regulation of muscle regeneration. Physiol. Rev. 84, 209-238. doi:10.1152/physrev.00019.2003

Ciciliot, S., and Schiaffino, S. (2010). Regeneration of mammalian skeletal muscle: basic mechanisms and clinical implications. Curr. Pharm. Des. 16, 906-914. doi:10.2174/138161210790883453

Couteaux, R., Mira, J. C., and D’Albis, A. (1988). Regeneration of muscles after cardiotoxin injury. I. Cytological aspects. Biol. Cell 62, 171-182. doi:10.1111/j. 1768-322X.1988.tb00719.x

De Luca, A., Nico, B., Liantonio, A., Didonna, M. P., Fraysse, B., Pierno, S., et al. (2005). A multidisciplinary evaluation of the effectiveness of cyclosporine a in dystrophic mdx mice. Am. J. Pathol. 166, 477-489. doi:10.1016/S0002-9440(10) 62270-5

Dunn, S. E., Simard, A. R., Prud'homme, R. A., and Michel, R. N. (2002). Calcineurin and skeletal muscle growth. Nat. Cell Biol. 4, E46. doi:10.1038/ncb0302-e46a author reply E46-47,

Grumati, P., Coletto, L., Sabatelli, P., Cescon, M., Angelin, A., Bertaggia, E., et al. (2010). Autophagy is defective in collagen VI muscular dystrophies, and its reactivation rescues myofiber degeneration. Nat. Med. 16, 1313-1320. doi:10.1038/ nm. 2247

Hawke, T. J., Meeson, A. P., Jiang, N., Graham, S., Hutcheson, K., DiMaio, J. M., et al. (2003). p21 is essential for normal myogenic progenitor cell function in regenerating skeletal muscle. Am. J. Physiol. Cell Physiol. 285, C1019-C1027. doi:10.1152/ajpcell.00055.2003

Homan, W. P., Fabre, J. W., Williams, K. A., Millard, P. R., and Morris, P. J. (1980). Studies on the immunosuppressive properties of cyclosporin A in rats receiving renal allografts. Transplantation 29, 361-366. doi:10.1097/00007890198005000-00003

Hudson, M. B., and Price, S. R. (2013). Calcineurin: a poorly understood regulator of muscle mass. Int. J. Biochem. Cell Biol. 45, 2173-2178. doi:10.1016/j.biocel. 2013.06.029

Hunt, J., Cheng, A., Hoyles, A., Jervis, E., and Morshead, C. M. (2010). Cyclosporin A has direct effects on adult neural precursor cells. J. Neurosci. 30, 2888-2896. doi:10.1523/JNEUROSCI.5991-09.2010

Irwin, W. A., Bergamin, N., Sabatelli, P., Reggiani, C., Megighian, A., Merlini, L., et al. (2003). Mitochondrial dysfunction and apoptosis in myopathic mice with collagen VI deficiency. Nat. Genet. 35, 367-371. doi:10.1038/ng1270
Lampe, A. K., and Bushby, K. M. D. (2005). Collagen VI related muscle disorders. J. Med. Genet. 42, 673-685. doi:10.1136/jmg.2002.002311

Liu, J., Farmer, J. D., Lane, W. S., Friedman, J., Weissman, I., and Schreiber, S. L. (1991). Calcineurin is a common target of cyclophilin-cyclosporin A and FKBPFK506 complexes. Cell 66, 807-815. doi:10.1016/0092-8674(91)90124-H

Merlini, L., Angelin, A., Tiepolo, T., Braghetta, P., Sabatelli, P., Zamparelli, A., et al. (2008). Cyclosporin A corrects mitochondrial dysfunction and muscle apoptosis in patients with collagen VI myopathies. Proc. Natl. Acad. Sci. U.S.A. 105, 5225-5229. doi:10.1073/pnas.0800962105

Merlini, L., Sabatelli, P., Armaroli, A., Gnudi, S., Angelin, A., Grumati, P., et al. (2011). Cyclosporine A in Ullrich congenital muscular dystrophy: long-term results. Oxid. Med. Cell. Longev. 2011, 139194. doi:10.1155/2011/139194

Michel, R. N., Dunn, S. E., and Chin, E. R. (2004). Calcineurin and skeletal muscle growth. Proc. Nutr. Soc. 63, 341-349. doi:10.1079/PNS2004362

Parsons, S. A., Millay, D. P., Sargent, M. A., Naya, F. J., McNally, E. M., Sweeney, H. L., et al. (2007). Genetic disruption of calcineurin improves skeletal muscle pathology and cardiac disease in a mouse model of limb-girdle muscular dystrophy. $J$. Biol. Chem. 282, 10068-10078. doi:10.1074/jbc.M609368200

Sakuma, K., Nakao, R., Aoi, W., Inashima, S., Fujikawa, T., Hirata, M., et al. (2005). Cyclosporin A treatment upregulates Id 1 and Smad 3 expression and delays skeletal muscle regeneration. Acta Neuropathol. 110, 269-280. doi:10.1007/s00401005-1049-x

Sakuma, K., Nishikawa, J., Nakao, R., Watanabe, K., Totsuka, T., Nakano, H., et al. (2003). Calcineurin is a potent regulator for skeletal muscle regeneration by association with NFATc1 and GATA-2. Acta Neuropathol. 105, 271-280. doi:10.1007/s00401-002-0647-0

Sakuma, K., and Yamaguchi, A. (2010). The functional role of calcineurin in hypertrophy, regeneration, and disorders of skeletal muscle. J. Biomed. Biotechnol. 2010, 721219. doi:10.1155/2010/721219

Schiaffino, S., and Serrano, A. L. (2002). Calcineurin signaling and neural control of skeletal muscle fiber type and size. Trends Pharmacol. Sci. 6147, 569-575. doi:10.1016/S0165-6147(02)02111-9

Serrano, A. L., Mann, C. J., Vidal, B., Ardite, E., Perdiguero, E., and Muñoz-Cánoves, P. (2011). Cellular and molecular mechanisms regulating fibrosis in skeletal muscle repair and disease. Curr. Top. Dev. Biol. 96, 167-201. doi:10.1016/B978-0-12385940-2.00007-3

Shi, X., and Garry, D. J. (2006). Muscle stem cells in development, regeneration, and disease. Genes Dev. 20, 1692-1708. doi:10.1101/gad.1419406

Stupka, N., Gregorevic, P., Plant, D. R., and Lynch, G. S. (2004). The calcineurin signal transduction pathway is essential for successful muscle regeneration in mdx dystrophic mice. Acta Neuropathol. 107, 299-310. doi:10.1007/s00401-0030807-x

Tedesco, F. S., Dellavalle, A., Diaz-Manera, J., Messina, G., and Cossu, G. (2010). Repairing skeletal muscle: regenerative potential of skeletal muscle stem cells. $J$. Clin. Invest. 120, 11-19. doi:10.1172/JCI40373

Urciuolo, A., Quarta, M., Morbidoni, V., Gattazzo, F., Molon, S., Grumati, P., et al. (2013). Collagen VI regulates satellite cell self-renewal and muscle regeneration. Nat. Commun. 4, 1964. doi:10.1038/ncomms2964

Zulian, A., Rizzo, E., Schiavone, M., Palma, E., Tagliavini, F., Blaauw, B., et al. (2014). NIM811, a cyclophilin inhibitor without immunosuppressive activity, is beneficial in collagen VI congenital muscular dystrophy models. Hum. Mol. Genet. doi: $10.1093 / \mathrm{hmg} / \mathrm{ddu} 254$

Conflict of Interest Statement: The authors declare that the research was conducted in the absence of any commercial or financial relationships that could be construed as a potential conflict of interest.

Received: 27 June 2014; accepted: 29 August 2014; published online: 15 September 2014. Citation: Gattazzo F, Molon S, Morbidoni V, Braghetta P, Blaauw B, Urciuolo A and Bonaldo P (2014) Cyclosporin A promotes in vivo myogenic response in collagen VIdeficient myopathic mice. Front. Aging Neurosci. 6:244. doi: 10.3389/fnagi.2014.00244 This article was submitted to the journal Frontiers in Aging Neuroscience.

Copyright $(2014$ Gattazzo, Molon, Morbidoni, Braghetta, Blaauw, Urciuolo and Bonaldo. This is an open-access article distributed under the terms of the Creative Commons Attribution License (CC BY). The use, distribution or reproduction in other forums is permitted, provided the original author(s) or licensor are credited and that the original publication in this journal is cited, in accordance with accepted academic practice. No use, distribution or reproduction is permitted which does not comply with these terms. 